\title{
Parasternal electromyography to determine the relationship between patient-ventilator asynchrony and nocturnal gas exchange during home mechanical ventilation set-up
}

\author{
Michelle Ramsay, ${ }^{1,2}$ Swapna Mandal, 1,2 Eui-Sik Suh, 1,2 Joerg Steier, 1,2 \\ Abdel Douiri, ${ }^{3,4}$ Patrick Brian Murphy, ${ }^{1}$ Michael Polkey, ${ }^{5}$ Anita Simonds, ${ }^{5}$ \\ Nicholas Hart ${ }^{1,2,4}$
}

- Additional material is published online only. To view please visit the journal online (http://dx.doi.org/10.1136/ thoraxjnl-2015-206944).

${ }^{1}$ Lane Fox Respiratory Unit, Guy's, St Thomas' NHS Foundation Trust, London, UK ${ }^{2}$ Division of Asthma, Allergy and Lung Biology, King's College London, London, UK ${ }^{3}$ Department of Public Health Sciences, King's College London, London, UK

${ }^{4}$ Guy's and St Thomas' NHS Foundation Trust and King's College London, National Institute Health Research Biomedical Research Centre, London, UK

${ }^{5}$ NIHR Respiratory Biomedical Research Unit, Royal Brompton \& Harefield NHS Foundation Trust and Imperial College, London, UK

\section{Correspondence to} Dr Michelle Ramsay, Lane Fox Unit, Ground Floor, St Thomas Hospital, Westminster Bridge Road, London SE1 7EH; UK: michelleramsay@doctors.org.uk

Received 15 February 2015 Revised 14 June 2015 Accepted 22 June 2015 Published Online First 21 July 2015
CrossMark

To cite: Ramsay $M$, Mandal S, Suh E-S, et al.

Thorax 2015;70:946-952.

\section{ABSTRACT}

Introduction Patient-ventilator asynchrony (PVA) can adversely affect the successful initiation of non-invasive home mechanical ventilation (HMV). The aim of this observational study was to quantify the prevalence of PVA during initiation of HMV and to determine the relationship between PVA and nocturnal gas exchange. Method Type and frequency of PVA were measured by surface parasternal intercostal muscle electromyography, thoracoabdominal plethysmography and mask pressure during initiation of HMV. Severe PVA was defined, as previously, as asynchrony affecting $\geq 10 \%$ of breaths. Results 28 patients (18 male) were enrolled aged $61 \pm 15$ years and with a body mass index of $35 \pm 9 \mathrm{~kg} / \mathrm{m}^{2}$. Underlying diagnoses were neuromuscular disease with or without chest wall disease $(n=6)$, obesity related chronic respiratory failure $(n=12)$ and COPD $(n=10)$. PVA was observed in all patients with $79 \%$ of patients demonstrating severe PVA. Triggering asynchrony was most frequent, observed in 24\% (IQR: $11-36 \%$ ) of breaths, with ineffective efforts accounting for 16\% (IQR: 4-24\%). PVA types were similar between disease groups, with the exception of auto-triggering, which was higher in patients with COPD (12\% (IQR: 6-26\%)). There was no correlation observed between PVA and time spent with oxygen saturations $\leq 90 \%$, mean oxygen saturations or transcutaneous carbon dioxide levels during overnight ventilation.

Conclusions Severe PVA was identified in the majority of patients, irrespective of pathophysiological disease state. This was not associated with ineffective ventilation as evidenced by gas exchange.

\section{INTRODUCTION}

A European survey performed in 2005 reported that almost 22000 patients with chronic respiratory failure were receiving home mechanical ventilation (HMV) with either non-invasive or invasive ventilation. ${ }^{1}$ Despite the clinical benefits, a significant proportion of patients are unable to adhere to their HMV prescription. ${ }^{2}$ Certain factors affecting adherence to HMV are difficult to modify, such as mask claustrophobia, but the clinical set-up of noninvasive ventilation (NIV) is operator-dependent

\section{Key messages}

What is the key question?

- To quantify the prevalence of patient-ventilator asynchrony (PVA) during home non-invasive ventilation set-up and to determine the relationship between PVA and nocturnal gas exchange?

\section{What is the bottom line?}

- PVA, measured using parasternal electromyography, is a very frequent event and, in contrast to current opinion, this study has shown that PVA was not associated with an adverse impact on nocturnal gas exchange.

\section{Why read on?}

- This physiological study of overnight set-up of non-invasive ventilation in patients with chronic respiratory failure using surface parasternal electromyography provides a comprehensive assessment of the type and frequency of PVA encountered when initiating home non-invasive ventilation.

and indeed could be enhanced if patient-ventilator synchrony were achieved.

Patient-ventilator asynchrony (PVA) describes the poor interaction between the patient and the ventilator and is the consequence of the respiratory muscle activity of the patient being opposed to the action of the ventilator. These are, in general, grouped as triggering and cycling PVA. PVA adversely effects respiratory muscle unloading during mechanical ventilation, ${ }^{3}{ }^{4}$ which is reported from previous studies to negatively impact on the nocturnal gas exchange and sleep quality. ${ }^{5-8}$ Furthermore, asynchronous breaths have previously been observed to have unfavourable clinical consequences including dyspnoea perception, ${ }^{9-13}$ patient discomfort and intolerance ${ }^{1415}$ and reduced adherence to NIV. ${ }^{16}$ Previous studies have reported that PVA can have an adverse clinical impact if greater than $10 \%$ of the breaths are asynchronous, ${ }^{14} 1718$ albeit a minimal clinically important difference 
with non-invasive ventilator support has yet to be reported. Furthermore, there are limited data on the type and prevalence of asynchronous events during NIV.

More recently, the surface parasternal electromyogram (sEMGpara) has been used for the non-invasive measurement of neural respiratory drive in acute and stable settings, including overnight measurements during sleep in patients with chronic respiratory disease. ${ }^{11-13}{ }^{19}$ As PVA describes the coordination between the respiratory effort of the patient and the mechanical breaths delivered by the ventilator, a real-time measurement of the relationship between neural respiratory drive during inspiration and the timing of ventilator pressurisation is required. We hypothesised, using this novel comparative technique, that we could quantify the type and prevalence of PVA in patients with chronic respiratory failure secondary to restrictive and obstructive lung disease during overnight HMV set-up and, in addition, investigated the relationship between PVA and nocturnal gas exchange.

\section{METHODS}

Patients

Patients were referred to a tertiary centre for management of chronic respiratory failure between September 2010 and December 2012. All participants provided written informed consent at enrolment.

\section{Definition of PVA}

Following a comprehensive review of the literature (see online supplementary material for search terms) and discussions with international HMV experts, a group consensus was reached in defining the types of PVA. We divided PVA into triggering and cycling asynchronies and used these as reference for this study. ${ }^{14} 172021$ The triggering asynchronies included ineffective efforts, auto-triggering, double triggering and multiple triggering and the cycling asynchronies included premature expiratory cycling, delayed expiratory cycling and autocycling. The definitions of the specific types of triggering and cycling PVA, with worked examples, are described in detail in the online supplementary appendix E1 and figure E1-E8.

\section{NIV set-up and measurements}

All patients were admitted for nocturnal set-up and titration of NIV (NIPPY3 + ventilator; B\&D Electromedical, Stratfordupon-Avon, UK) according to standard clinical protocols depending upon their underlying pathophysiological condition (see online supplementary appendix E2a-E2c) in a pressure support mode with a fixed backup rate set at two breaths below the resting breathing rate, as previously reported. ${ }^{22}$ Measurement of sEMGpara was employed as a physiological biomarker to mark the onset and level of neural respiratory drive. ${ }^{11-13}$ The second intercostal space was identified using bony landmarks and skin was prepared with EMG contact gel (Nuprep, DO Weaver and Co, Colorado, USA). Wet gel electrodes (Neuroline 720, Ambu, Ballerup, Denmark) were placed adjacent to the sternal edge in the second intercostal spaces. The sEMGpara signal was amplified, processed, filtered and analysed in standard conditions according to the methods previously described $^{12}$ (see online supplementary material for details). sEMGpara recordings were performed with the patient lying in bed in their preferred sleeping position and acquired throughout the whole night. Respiratory inductance plethysmography (Respitrace, Sensormedics, Miami Beach, Florida) was concurrently used to measure chest wall excursion and abdominal wall excursion to assist the analyser to gait against postural sEMGpara movement artefacts. Patients underwent overnight measurements of mask pressure using a standard pressure transducer with a range between $+200 \mathrm{~cm} \mathrm{H}_{2} \mathrm{O}$ and $-200 \mathrm{~cm} \mathrm{H}_{2} \mathrm{O}$ (GM Instruments, Kilwinning, UK). Transcutaneous oximetry $\left(\mathrm{SpO}_{2}\right)$ and capnometry $\left(\mathrm{TcCO}_{2}\right)$ were calibrated to arterial carbon dioxide levels and measured using a TOSCA 500 monitor (Radiometer Medical, Copenhagen, Denmark). All data were analysed using LabChart V7.3.7 (AD Instruments, Chalgrove, UK) (figure 1).

\section{Type and frequency of PVA}

Manual recording analysis was performed in $10 \mathrm{~min}$ epochs throughout the whole night of NIV use, with the final 2 min of each epoch analysed for type and frequency of PVA according to the a priori definitions (see online supplementary appendix $\mathrm{E} 1$ and figures E1-E8). The 1st hour of recording and movement artefacts were discarded with the nearest artefact-free 2 min epoch analysed instead. The proportion of PVA was calculated as the number of asynchronous breaths divided by the total number of breaths (requested and delivered) multiplied by 100 , as described by Thille et al. ${ }^{17}$

\section{Ventilator set-up}

Every ventilator-delivered breath in the 10 min epochs was analysed as a triggered pressure support delivered breath, an inappropriate auto-triggered delivered breath or an appropriate pressure control delivered 'backup' breath (identified by rate and set inspiratory time). All ventilators were set in pressure support $(\mathrm{S} / \mathrm{T})$ mode with a backup rate set 2 below the resting respiratory rate. Comparative analysis of the PVA frequency when patients received $\geq 70 \%$ breaths from either pressure supported (patient triggered) or pressure controlled (ventilator delivered) ventilation was performed.

\section{Inter-rater reliability of PVA}

Two assessors (authors MR and SM) were blinded and independently scored 10 randomly selected $1 \mathrm{~h}$ sections of recorded data from 10 patients. Asynchronous events were manually assessed according to the a priori definitions (see online supplementary appendix E1 and figures E1-E8).

\section{Statistical analysis}

Patient demographic data and the type of ventilator-delivered breaths are expressed as mean $\pm \mathrm{SD}$, and a one-way analysis of variance and pairwise tests with a Bonferroni correction were used to compare patient groups. Data that was not normally distributed are reported as median values with IQRs. Comparative analysis of the differences in the ventilator set-up parameters and frequency of the types of PVA between the patient groups were made using the Kruskal-Wallis test. Comparative analysis between the number of pressure support breaths and pressure control breaths delivered by the ventilator were assessed using a Mann-Whitney U test. Simple rank regressions were performed to assess the strength of the relationship with PVA and nocturnal gas exchange. Statistical analyses were conducted using SPSS (V.19, IBM Corporation, USA) and GraphPad (Prism 6, GraphPad software, USA). A p value $<0.05$ was taken to represent statistical significance. Further details of the statistical analyses performed are placed in the online supplementary material.

\section{RESULTS}

Type and frequency of PVA

Patient demographics

All 28 patients (18 male) approached were recruited at an age of $61 \pm 15$ years, a body mass index (BMI) of $35 \pm 9 \mathrm{~kg} / \mathrm{m}^{2}$, an 


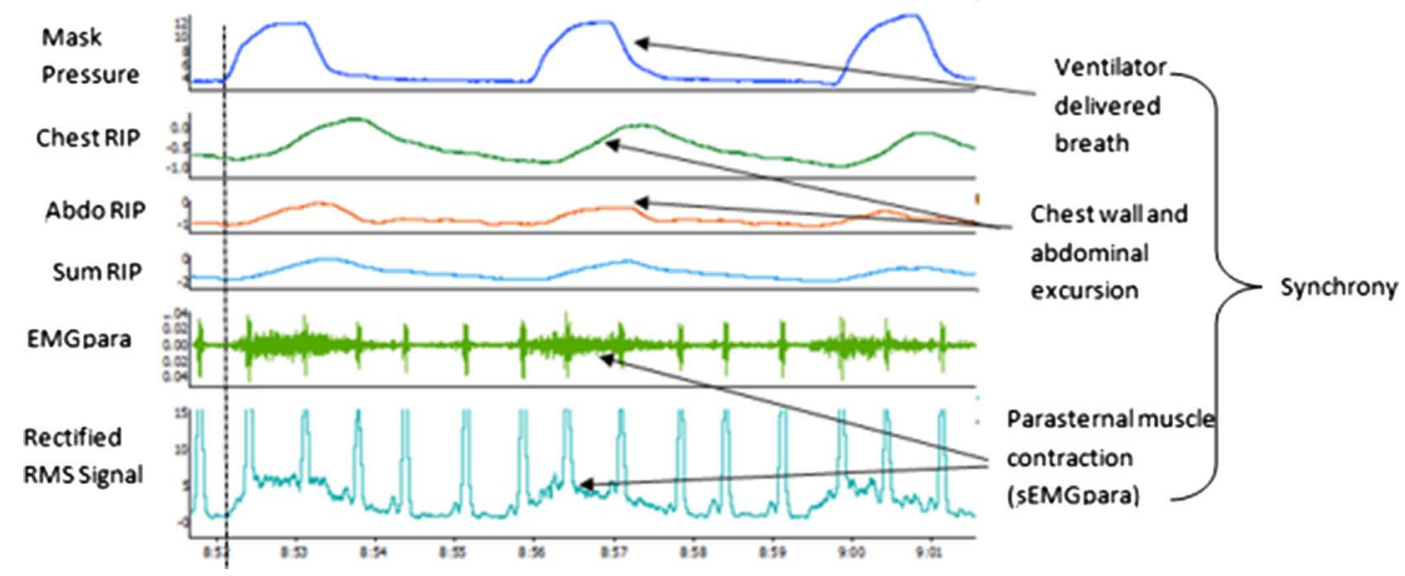

Figure 1 An example of satisfactory patient ventilator synchrony. Chest RIP, chest wall respiratory inductance plethysmography; Abdo RIP, abdominal respiratory inductance plethysmography; Sum RIP, combined chest and abdominal inductance plethysmography; EMGpara, parasternal intercostal electromyography; RMS, rectified root mean square of parasternal electromyography signal.

$\mathrm{FEV}_{1}$ of $1.1 \pm 0.5 \mathrm{~L}$ and an FVC of $1.6 \pm 0.7 \mathrm{~L}$. Six patients had neuromuscular disease with or without chest wall disease (NMD-CWD), 12 had obesity related respiratory failure (ORRF; BMI $\geq 30 \mathrm{~kg} / \mathrm{m}^{2}$ with a daytime $\mathrm{pCO}_{2} \geq 6 \mathrm{kPa}$ in the absence of any other cause) and 10 had COPD. Baseline demographic data are presented in table 1.

There were no differences in the age of the patients between patient groups and all patients had a BMI in the 'overweight' or 'obese' range. Four of the 10 patients with COPD had concurrent upper airways obstruction defined by a baseline oxygen desaturation index below $4 \%$ above 10 events per hour reflecting increasing trends of obesity in the UK. Patients with COPD were also more hypoxic and hypercapnic than the patients with NMD-CWD at baseline (arterial oxygen level $7.6 \pm 1.4 \mathrm{kPa}$ vs $9.2 \pm 0.6 \mathrm{kPa} ; \mathrm{p}<0.04$ and arterial carbon dioxide level 7.9 $\pm 1.0 \mathrm{kPa}$ vs $6.6 \pm 1.0 \mathrm{kPa}$; $<<0.03$, respectively).

\section{Ventilator set-up}

There was no difference between the number of pressure supported and pressure controlled breaths delivered overnight across the whole group $(44 \pm 26 \%$ vs $41 \pm 31 \% ; \mathrm{p}=0.7)$. Individual disease groups exhibited different interactions with the ventilator in terms of triggering the ventilator (table 2). Furthermore, as expected, those patients from the cohort that received over $70 \%$ pressure support breaths $(n=6)$ had a higher percentage of asynchronous breaths overnight than those patients that received over $70 \%$ pressure control breaths $(n=5)$ (30\% (27-38\%) vs 9\% (1-10\%), respectively; $\mathrm{p}<0.01)$. Patients that received more than $70 \%$ pressure support breaths demonstrated higher levels of ineffective efforts than those with more than $70 \%$ of pressure control delivered breaths (15\% (10-20\%) vs $1 \%(0-2 \%) ; \mathrm{p}<0.01)$.

Further details of the ventilator settings used between patient groups is available in the online supplementary material results section.

\section{Patient-ventilator asynchrony}

A total of 27637 breaths were analysed from the 2 min epochs of overnight data recorded. Combined triggering and cycling PVA had an overall prevalence of $28 \pm 19 \%$ across all patients studied. Indeed, $79 \%$ of patients were observed to have an asynchrony index greater than $10 \%$ of breaths (range 11-63\%). Ineffective efforts were the most common PVA observed in $89.3 \%$ of patients and accounted for $15 \pm 11 \%$ of breaths. Other PVA including auto-triggering, delayed expiratory cycling and premature cycling were observed in $85.7 \%, 67.9 \%$ and $85.7 \%$ of patients but with only $3 \%(0-9 \%), 1 \%(0-5 \%)$ and $1 \%(0-2 \%)$ of the total breaths affected, respectively. Double triggering, multiple triggering and autocycling were less frequent affecting $53.6 \%, 35.7 \%$ and $46.4 \%$ of patients,

Table 1 Comparison of baseline patient demographics by disease group

\begin{tabular}{|c|c|c|c|c|}
\hline Demographics & $\begin{array}{l}\text { COPD } \\
(n=10)\end{array}$ & $\begin{array}{l}\text { NMD-CWD } \\
(n=6)\end{array}$ & $\begin{array}{l}\text { ORRF } \\
(n=12)\end{array}$ & $\mathrm{p}$ Value \\
\hline Age (years) & $66 \pm 10$ & $58 \pm 22$ & $59 \pm 16$ & 0.54 \\
\hline BMI $\left(\mathrm{kg} / \mathrm{m}^{2}\right)$ & $30.0 \pm 5.2$ & $27.2 \pm 5.3$ & $43.0 \pm 4.8$ & $<0.0001^{*}$ \\
\hline $\begin{array}{l}\mathrm{FEV}_{1}(\% \\
\text { predicted) }\end{array}$ & $31.1 \pm 11.3$ & $32.7 \pm 11.3$ & $52.5 \pm 20.2$ & $<0.01^{*}$ \\
\hline FVC (\% predicted) & $52.4 \pm 9.7$ & $30.5 \pm 10.7$ & $55.6 \pm 19.0$ & $<0.01^{*}$ \\
\hline $\mathrm{FEV}_{1} / \mathrm{FVC}$ (\% ratio) & $45.8 \pm 11.0$ & $85.4 \pm 3.6$ & $81.2 \pm 8.5$ & $<0.001^{*}$ \\
\hline $\mathrm{PaO}_{2}(\mathrm{kPa})$ & $7.6 \pm 1.4$ & $9.2 \pm 0.6$ & $8.3 \pm 1.0$ & $<0.03^{*}$ \\
\hline $\mathrm{PaCO}_{2}(\mathrm{kPa})$ & $7.9 \pm 1.0$ & $6.6 \pm 1.0$ & $7.1 \pm 0.9$ & $<0.04^{*}$ \\
\hline $\mathrm{HCO}_{3}^{-}(\mathrm{mmols} / \mathrm{l})$ & $33.5 \pm 3.2$ & $30.8 \pm 3.8$ & $31.5 \pm 3.8$ & 0.28 \\
\hline
\end{tabular}

${ }^{*} \mathrm{~A}$ one-way analysis of variance was used to compare the demographics between disease groups, $\mathrm{p}$ value $<0.05$ was taken to represent statistical significance.

$\mathrm{BMI}$, body mass index; $\mathrm{HCO}_{3}^{-}$, serum bicarbonate level; $\mathrm{NMD}-\mathrm{CWD}_{\text {, neuromuscular disease with or without chest wall disease; ORRF, obesity related respiratory failure; PaCO }}$, arterial carbon dioxide level; $\mathrm{PaO}_{2}$, arterial oxygen level. 
Table 2 Comparison of non-invasive ventilator set-up by disease group

\begin{tabular}{|c|c|c|c|c|}
\hline Ventilator parameters & COPD & NMD-CWD & ORRF & p Value \\
\hline IPAP $\left(\mathrm{cmH}_{2} \mathrm{O}\right)$ & $18(18-25)$ & $15(14-21)$ & $20(18-28)$ & 0.1 \\
\hline $\operatorname{EPAP}\left(\mathrm{cmH}_{2} \mathrm{O}\right)$ & $4(3-7)$ & $3(3-4)$ & $9(8-12)$ & $<0.001^{*}$ \\
\hline Backup rate (breaths/min) & $14(11-14)$ & $14(14-14)$ & $14(13-16)$ & 0.3 \\
\hline$\%$ Pressure support breaths & $60 \pm 25$ & $34 \pm 24$ & $36 \pm 56$ & 0.06 \\
\hline$\%$ Pressure control breaths & $25 \pm 30$ & $47 \pm 24$ & $51 \pm 31$ & 0.09 \\
\hline$\%$ Ineffective efforts & $15 \pm 11$ & $19 \pm 13$ & $13 \pm 10$ & 0.4 \\
\hline$\%$ Total patient ventilator asynchrony & $43 \pm 29$ & $29 \pm 19$ & $24 \pm 17$ & 0.3 \\
\hline
\end{tabular}

respectively, and these accounted for $0 \%(0-1 \%), 0 \%(0-0) \%$ and $0 \%(0-0 \%)$ of breaths, respectively.

\section{Comparison of PVA by disease category}

Across all groups, triggering asynchronies were greater than cycling asynchronies with 24\% (11-36\%) of total breaths demonstrating triggering asynchronies and 5\% (1-8\%) of total breaths demonstrating cycling asynchronies in all patients $(\mathrm{p}<0.0001)$. PVA, including triggering asynchronies (table 3 ) and cycling asynchronies (table 4), was observed in all patient groups ranging from $33 \%(19-78 \%)$ of total breaths in patients with COPD, 31\% (10-45\%) of breaths in patients with NMD-CWD and 29\% (9-38\%) of breaths in patients with ORRF $(p=0.3)$.

The most frequent asynchrony was ineffective effort, albeit there was no difference across the disease groups $(p=0.4)$. The only difference observed between patients with COPD, NMD-CWD and ORRF was in auto-triggering, affecting 12\% (6-36\%), 6\% (1-8\%) and $2 \%(0-6 \%)$ of breaths, respectively $(p=0.04)$.

\section{Overnight gas exchange}

Overnight gas exchange was assessed using nocturnal oximetry and capnography, which has been shown to accurately monitor and track changes in nocturnal gas exchange in patients receiving NIV. ${ }^{23}$ Patients demonstrated nocturnal hypoventilation with a mean $\mathrm{SpO}_{2}$ of $91 \pm 7 \%$ and $\mathrm{TcCO}_{2}$ of $7.8 \pm 1.3 \mathrm{kPa}$ during the first night of NIV set-up without the use of supplemental oxygen. Of the overnight total analysis time $35 \pm 33 \%$ was spent with oxygen saturations below 90\% (TBI90) in a subset of 16 patients. Patients with ORRF were more hypoxic overnight than patients with NMD-CWD $\left(\mathrm{SpO}_{2}\right.$ of $88 \pm 8 \%$ vs $96 \pm 4 \%$; $\mathrm{p}=0.02)$. There were no associations observed between total overnight PVA events and nocturnal $\mathrm{SpO}_{2}\left(\mathrm{r}^{2}=0.08 ; \mathrm{p}=0.16\right)$ and $\mathrm{TcCO}_{2}\left(\mathrm{r}^{2}<0.001 ; \mathrm{p}=0.94\right)$. Furthermore, there was no relationship observed between nocturnal $\mathrm{SpO}_{2}$ and $\mathrm{TcCO}_{2}$ and the percentage of ineffective efforts $\left(r^{2}=0.04 ; p=0.32\right.$ and $r^{2}=-0.04 ; p=0.32$, respectively). Finally, there was no association observed between the TBI90 and the percentage of asynchronous breaths or ineffective efforts $\left(\mathrm{r}^{2}=-0.02 ; \mathrm{p}=0.12\right.$ and $\mathrm{r}^{2}=-0.08 ; \mathrm{p}=0.36$, respectively) in the subset of patients examined.

\section{Inter-rater reliability of assessment of PVA}

Ten patients (four COPD, four ORRF and two NMD-CWD) were included in this substudy. A total of 4603 breaths were analysed by two independent scorers with PVA reported in 812 (35\%) and 891 (39\%) breaths, respectively. The intraclass correlation coefficient between the scorers was $0.84(0.74-0.90)$. The predominant asynchrony reported was ineffective efforts, for which there was the closest interobserver agreement with an intraclass correlation coefficient of 0.94 (0.79-0.99). The agreement for triggering and cycling PVA are shown in tables 5 and 6. The lowest agreement was observed in the autocycling asynchrony $(0.22 ;-0.54-0.74)$, albeit this had a very low frequency and was observed in $<1 \%$ of the total breaths analysed.

\section{DISCUSSION}

This is the first study to report a comprehensive overnight assessment of PVA during first night initiation of non-invasive ventilation. Previous studies have either been daytime or overnight studies in patients established on HMV and these studies lacked description of all types and frequencies of PVA. ${ }^{6-8} 141724$ Neural respiratory drive, as measured from surface electromyogram of the second intercostal parasternal muscles, combined with
Table 3 Triggering asynchrony during overnight NIV setup

\begin{tabular}{lcccl}
\hline$\%$ of breaths & COPD & NMD-CWD & ORRF & p Value \\
\hline \% Ineffective efforts & $15(6-23)$ & $24(5-27)$ & $14(2-21)$ & 0.4 \\
$\%$ Auto-triggering & $12(6-36)$ & $2(0-6)$ & $6(1-8)$ & $0.04^{*}$ \\
\% Multiple triggering & $0(0-0)$ & $0(0-0)$ & $0(0-0)$ & 0.1 \\
\% Double triggering & $0(0-1)$ & $0(0-3)$ & $0(0-1)$ & 0.6 \\
$\%$ Total triggering asynchrony & $26(13-66)$ & $27(7-39)$ & $21(9-31)$ & 0.3 \\
\hline
\end{tabular}

A Kruskal-Wallis test was used to compare patient-ventilator asynchrony between disease groups, $\mathrm{p}$ value $<0.05$ was taken to represent statistical significance. NMD-CWD, neuromuscular disease with or without chest wall disease; ORRF, obesity related respiratory failure.
Table 4 Cycling asynchrony during overnight NIV setup

\begin{tabular}{lllll}
\hline \% of breaths & COPD & NMD-CWD & ORRF & p Value \\
\hline \% Premature expiratory cycling & $1(0-4)$ & $1(0-4)$ & $1(0-2)$ & 0.9 \\
\% Delayed expiratory cycling & $2(0-7)$ & $2(0-5)$ & $0(0-3)$ & 0.5 \\
\% Auto-cycling & $1(0-4)$ & $0(0-0)$ & $0(0-0)$ & 0.2 \\
$\%$ Total cycling asynchrony & $7(4-10)$ & $4(3-6)$ & $3(1-7)$ & 0.2 \\
\hline
\end{tabular}

A Kruskal-Wallis test was used to compare patient-ventilator asynchrony between disease groups, $p$ value $<0.05$ was taken to represent statistical significance. NMD-CWD, neuromuscular disease with or without chest wall disease; ORRF, obesity related respiratory failure. 
Table 5 Agreement of triggering asynchrony between two independent scorers

\begin{tabular}{llccc}
\hline Type of asynchrony & Scorer $\mathbf{1} \mathbf{n}$ (\% breaths) & Scorer $\mathbf{~ n}$ (\% breaths) & ICC (95\% Cl) & $\begin{array}{l}\text { Bland-Altman analysis } \\
\text { bias (95\% limits of agreement) }\end{array}$ \\
\hline Ineffective efforts & $484(21 \%)$ & $466(21 \%)$ & $0.94(0.79$ to 0.99$)$ & $1.8(-30.1$ to 33.7$)$ \\
Auto-triggering & $164(7 \%)$ & $231(10 \%)$ & $0.77(0.19$ to 0.94$)$ & $-6.7(-25.4$ to 12.0$)$ \\
Double triggering & $22(<1 \%)$ & $11(<1 \%)$ & $0.67(0.11$ to 0.91$)$ & $1.1(-1.9$ to 4.1$)$ \\
Multiple triggering & $4(<1 \%)$ & $4(<1 \%)$ & 1.00 & $0.0(0.0$ to 0.0$)$ \\
\hline
\end{tabular}

ICC, intraclass correlation coefficient.

measurements of thoracoabdominal motion and mask pressure has been shown to be accurate and reliable in assessing trigger and cycling PVA during NIV. Importantly, we have detailed the high prevalence of PVA during initiation of HMV in patients with stable chronic respiratory failure and shown that up to a third of the breaths were asynchronous during the initiation of HMV, irrespective of the underlying disease pathology. Triggering asynchrony rather than cycling asynchrony was shown to be the most frequent PVA. Of major relevance to the clinician, there was no relationship between PVA and overnight gas exchange, which implies that the adequate delivery of ventilation during HMV set-up, based on the frequency and level of airway pressurisation, is of greater importance to optimise adequacy of gas exchange, rather than optimising PVA.

\section{Critique of the method}

We acknowledge that sEMGpara has recognised limitations, including postural artefacts and potential contamination of the signal from other chest wall muscles. ${ }^{25}$ Needle electrodes could have been used to directly isolate parasternal muscle activity, but this is unsuitable for overnight monitoring in the clinical setting and may risk pneumothorax. ${ }^{26}$ Alternatively, an oesophageal electrode may be used to monitor the diaphragm electromyogram, but again this is poorly tolerated in stable patients and the use of such an invasive technique in clinical practice for overnight studies is wholly challenging.

Although patients in this study were directly observed to be sleeping overnight by the research team, this was not confirmed through full montage polysomnography (PSG) and the authors acknowledge this. However, PSG is not routinely used in clinical practice for $\mathrm{HMV}$ initiation in the UK, and indeed recent European guidance has recommended using PSG only when simple tools to optimise NIV set-up have failed. ${ }^{27}$

For practical reasons, and based on our previous daytime laboratory studies, ${ }^{28}$ mask leak was not assessed in this overnight study as the burden of the pneumotach in the experimental mask-ventilator circuit actually contributed to greater air leak and consequently this would have increased PVA. Indeed, air leak induced PVA would have been a major confounder during the overnight studies. ${ }^{7} 14$

\section{Physiological observations}

\section{Prevalence of PVA}

By using a non-invasive approach to monitor physiological variables over the whole night, we have demonstrated that the prevalence of PVA, irrespective of the underlying disease pathology, was substantially greater than that previously reported. $^{6-8} 1417{ }^{24} \mathrm{Up}$ to a third of all breaths, during NIV set-up, were asynchronous. PVA was demonstrated in all patients studied, compared with previous studies which have reported a PVA prevalence of $0-77 \%$ of patients. ${ }^{5} 24$ These current data reflect the enhanced details of this advanced monitoring technique.

\section{Triggering asynchrony}

An ineffective effort was the most frequent type of PVA observed during the initiation of HMV. Similar to recent work by Carlucci et al, ${ }^{16}$ there was no difference in the prevalence of this asynchrony between the patient groups. Interestingly, autotriggered breaths affected most patients, but the frequency of these events was very low. Furthermore, auto-triggering was most commonly observed in patients with COPD and this is likely to be a consequence of mask leak, as previously reported. ${ }^{24}$ In addition, patients with COPD have an increased airway secretion load and this has previously been demonstrated to contribute to auto-triggering of the ventilator. ${ }^{29}$ Despite the observation that double triggering and multiple triggering were demonstrated in up to $50 \%$ of patients, the frequency of this PVA in the current study was very low and therefore unlikely to have clinical relevance during the initiation of HMV. Furthermore, cycling asynchrony was observed to be infrequent.

\section{Asynchrony and overnight gas exchange}

Despite higher levels of asynchrony observed in our cohort, there was no demonstrable effect on overnight gas exchange. This is in contrast to previous data suggesting that ineffective efforts are associated with overnight oxygen desaturation. ${ }^{6}$ This may, in part, be explained by the ventilator backup rate in the current study, supported by the proportion of pressure controlled breaths delivered, which was up to $46 \%$, depending on the patient group. The study by Fanfulla et $a l^{6}$ was performed

Table 6 Agreement of cycling asynchrony between two scorers

\begin{tabular}{|c|c|c|c|c|}
\hline Type of asynchrony & $\begin{array}{l}\text { Scorer } 1 \\
\text { n (\% breaths) }\end{array}$ & $\begin{array}{l}\text { Scorer } 2 \\
\text { n (\% breaths) }\end{array}$ & ICC (95\% CI) & $\begin{array}{l}\text { Bland-Altman analysis } \\
\text { bias ( } 95 \% \text { limits of agreement) }\end{array}$ \\
\hline Premature cycling & $88(4 \%)$ & $124(5 \%)$ & 0.73 (0.26 to 0.92$)$ & $-3.6(-18.7$ to 11.5$)$ \\
\hline Extended cycling & $45(2 \%)$ & $50(2 \%)$ & $0.76(0.31$ to 0.93$)$ & -0.5 (-8.6 to 7.6$)$ \\
\hline $\begin{array}{l}\text { Auto-cycling } \\
\text { cycling }\end{array}$ & $5(<1 \%)$ & $5(<1 \%)$ & $0.22(-0.54$ to 0.74$)$ & $0.0(-2.3$ to 2.3$)$ \\
\hline
\end{tabular}


in patients already established on HMV and the study used low level pressure support and, rather unusually for patients with NMD, there was no back-up rate applied. This would not be the current clinical approach, based on the current evidence, which supports the use of a mandatory backup rate in patients with $\mathrm{NMD}^{30}$ patients with $\mathrm{ORRF}^{22} 31$ and patients with COPD. ${ }^{32}$ The current data have demonstrated that optimal set-up of the inspiratory positive airway pressure, the expiratory positive airway pressure and the backup rate is more important, in terms of control of gas exchange, than PVA.

\section{Clinical implications}

Previous work has shown that clinician scoring of PVA through non-invasive measurements of ventilator flow and pressure waveform fail to report up to two-thirds of events. ${ }^{33}$ Other studies using automated algorithms to detect asynchrony have improved frequency detection but have been limited in detailing the type of PVA. ${ }^{34} 35$ However, the current study has demonstrated adequate levels of agreement between two independent observers comprehensively reporting triggering and cycling asynchrony. This non-invasive technique is potentially suitable to assist the clinician in optimising the ventilator settings during NIV set-up with real-time visual feedback of the impact of the ventilator changes at the bedside. For example, this novel approach can determine the physiological cause of the double triggered breath, where other methods would fail. This event can occur due to the first ventilator-delivered breath prematurely cycling out and the second ventilator-delivered breath appropriately triggered by the ongoing inspiratory effort of the patient, evidenced by increasing amplitude of the neural respiratory drive (figure 2A). Alternatively, this event can reflect inappropriate auto-triggering of the second breath such that there is no increase in the neural respiratory drive observed (figure 2B). The addition of sEMGpara, as a marker of neural inspiratory drive, assists the scorer in distinguishing between the two phenomena and enables appropriate adjustment of the ventilator.

Previous work has suggested that PVA, in particular ineffective efforts, has an adverse effect of overnight gas exchange. ${ }^{6}$ However, the data from the current study have shown that during NIV set-up PVA has limited impact on gas exchange, in terms of $\mathrm{SpO}_{2}$, TBI90 and $\mathrm{TcCO}_{2}$ levels. Even high levels of asynchrony, irrespective of the type, had limited effect on overnight gas exchange, suggesting that adequate ventilation, based on inspiratory and expiratory positive airway pressures and backup rate, will enhance gas exchange irrespective of patientventilator synchronisation. Furthermore, the current study indicates that optimising the ventilator settings so that the patient receives a greater proportion of pressure controlled breaths than pressure supported breaths may confer a benefit in reducing levels of PVA and, in particular, ineffective efforts. This is supported by previous data published by our own group on morbidly obese patients ${ }^{22}$ and it may explain, in part, the benefits of a high intensity non-invasive positive pressure ventilation in stable COPD. ${ }^{32}$ Controlled data detailing the effect of PVA on patient-centred outcomes, such as sleep quality, daytime symptoms and health related quality of life, will be reported in the future (http://www.clinicaltrials.gov NCT01371149).

\section{CONCLUSION}

Physiological measurements combining neural respiratory drive, thoracoabdominal movement and mask pressure monitoring

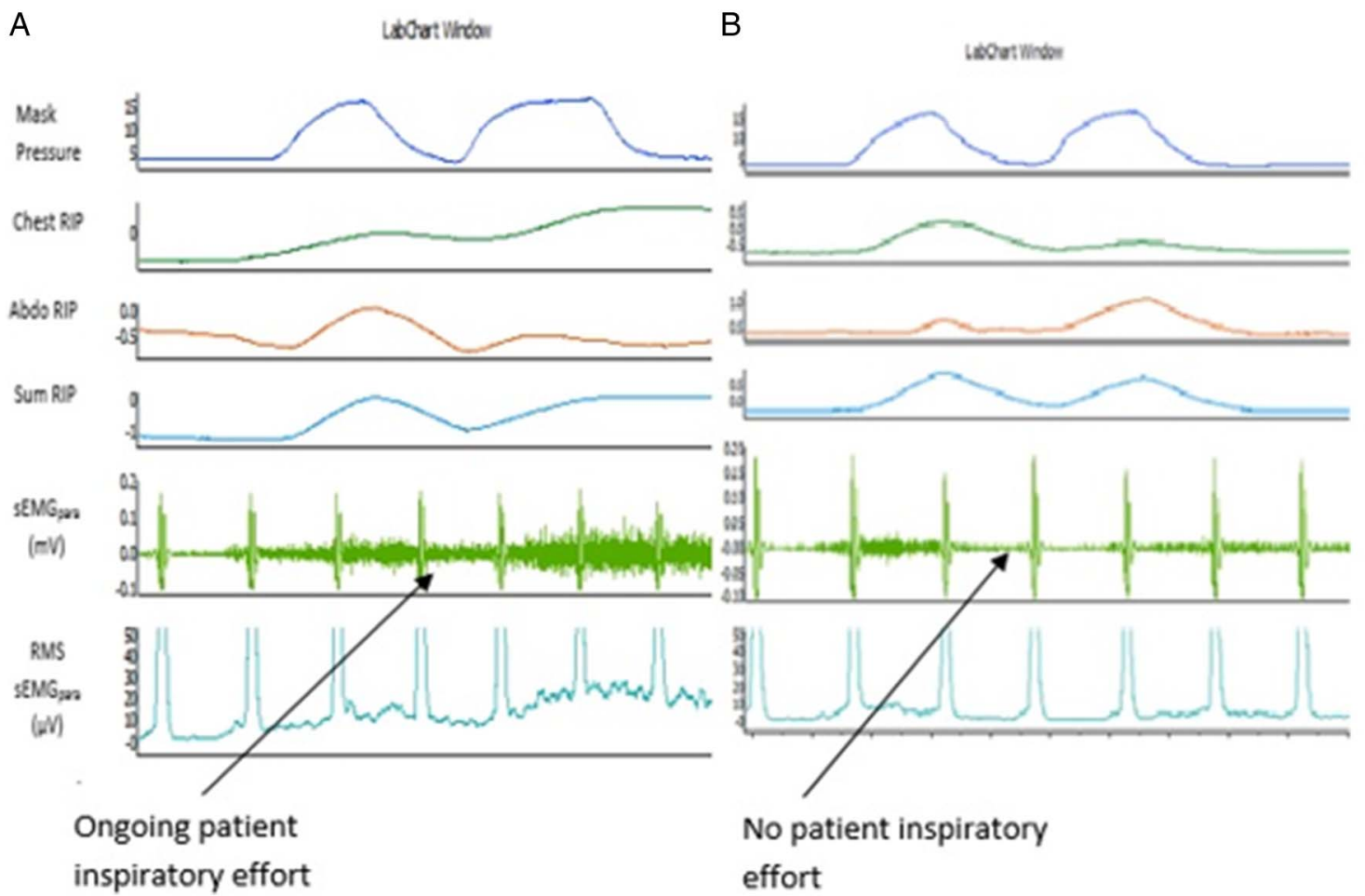

Figure 2 'Double triggering' was confirmed with the addition of second intercostal space electromyography to the mask pressure measurement and respiratory inductance plethysmography. (A) shows a prematurely cycled breath with ongoing patient inspiratory effort to trigger a second ventilator-delivered breath and (B) shows a patient-triggered breath followed by a nonpatient-triggered breath, which by definition is an 'auto-triggered' breath. Chest RIP, chest wall respiratory inductance plethysmography; Abdo RIP, abdominal respiratory inductance plethysmography; Sum RIP, combined chest and abdominal inductance plethysmography; EMGpara, parasternal intercostal electromyography; RMS, rectified root mean square of parasternal electromyography signal. 
have been shown as a reliable technique to non-invasively score the type and frequency of PVA observed during initiation of NIV in patients with chronic respiratory failure. Using this technique, we have demonstrated high levels of asynchrony, irrespective of the underlying aetiology of chronic respiratory failure, with triggering asynchrony reported as the most frequent type of asynchrony observed. Of major clinical relevance, there was no correlation between the type and frequency of asynchrony and overnight gas exchange during inpatient NIV set-up.

Contributors $\mathrm{NH}, \mathrm{AS}, \mathrm{PBM}, \mathrm{MR}$ and SM: contributed to the conception and design of this study. MR, SM and E-SS: assisted with the acquisition of data in this study. MR, SM, JS, PBM, AS and NH: contributed to the interpretation of data. MR, SM, AD, PBM and NH: assisted with statistical analysis of data. MR and SM: wrote the first draft of the paper and made all subsequent amendments. PBM, MP, JS, E-SS, AS and NH: critically appraised, revised the manuscript and assisted with producing the final version. All authors were in agreement with the final version of the manuscript submitted to Thorax. MR and NH will act as guarantors for the work.

Funding Guy's and St Thomas' Charity (Grant no. G100103).

Competing interests None declared.

Ethics approval National research ethics committee for human research.

Provenance and peer review Not commissioned; externally peer reviewed.

Data sharing statement Additional unpublished data regarding follow-up, sleep quality, respiratory muscle strength, quality of life questionnaires will be available as part of a PhD thesis.

\section{REFERENCES}

1 Lloyd-Owen SJ, Donaldson GC, Ambrosino N, et al. Patterns of home mechanical ventilation use in Europe: results from the Eurovent survey. Eur Respir J 2005;25:1025-31.

2 Criner GJ, Brennan K, Travaline JM, et al. Efficacy and compliance with noninvasive positive pressure ventilation in patients with chronic respiratory failure. Chest 1999;116:667-75.

3 Leung P, Jubran A, Tobin MJ. Comparison of assisted ventilator modes on triggering, patient effort, and dyspnea. Am J Respir Crit Care Med 1997;155:1940-8.

4 Nava S, Bruschi C, Fracchia C, et al. Patient-ventilator interaction and inspiratory effort during pressure support ventilation in patients with different pathologies. Eur Respir J 1997;10:177-83.

5 Fanfulla F, Delmastro M, Berardinelli A, et al. Effects of different ventilator settings on sleep and inspiratory effort in patients with neuromuscular disease. Am J Respir Crit Care Med 2005;172:619-24.

6 Fanfulla F, Taurino AE, Lupo ND, et al. Effect of sleep on patient/ventilator asynchrony in patients undergoing chronic non-invasive mechanical ventilation. Respir Med 2007;101:1702-7.

7 Crescimanno G, Canino M, Marrone 0. Asynchronies and sleep disruption in neuromuscular patients under home noninvasive ventilation. Respir Med 2012;106:1478-85.

8 Guo YF, Sforza E, Janssens JP. Respiratory patterns during sleep in obesity-hypoventilation patients treated with nocturnal pressure support: a preliminary report. CHEST 2007;131:1090-9.

9 Adler D, Perrig S, Takahashi H, et al. Polysomnography in stable COPD under non-invasive ventilation to reduce patient-ventilator asynchrony and morning breathlessness. Sleep Breath 2012;16:1081-90.

10 Jolley CJ, Moxham J. A physiological model of patient-reported breathlessness during daily activities in COPD. Eur Respir Rev 2009;18:66-79.

11 Reilly CC, Ward K, Jolley CJ, et al. Neural respiratory drive, pulmonary mechanics and breathlessness in patients with cystic fibrosis. Thorax 2011;66:240-6.
12 Murphy PB, Kumar A, Reilly C, et al. Neural respiratory drive as a physiological biomarker to monitor change during acute exacerbations of COPD. Thorax 2011;66:602-8.

13 Steier J, Jolley CJ, Polkey MI, et al. Nocturnal asthma monitoring by chest wall electromyography. Thorax 2011;66:609-14.

14 Vignaux L, Vargas F, Roeseler J, et al. Patient-ventilator asynchrony during non-invasive ventilation for acute respiratory failure: a multicenter study. Intensive Care Med 2009:35:840-6.

15 Vitacca M, Bianchi L, Zanotti E, et al. Assessment of physiologic variables and subjective comfort under different levels of pressure support ventilation. Chest 2004;126:851-9.

16 Carlucci A, Pisani L, Ceriana P, et al. Patient-ventilator asynchronies: may the respiratory mechanics play a role? Crit Care 2013;17:R54.

17 Thille AW, Rodriguez P, Cabello B, et al. Patient-ventilator asynchrony during assisted mechanical ventilation. Intensive Care Med 2006;32:1515-22.

18 de Wit M, Miller KB, Green DA, et al. Ineffective triggering predicts increased duration of mechanical ventilation. Crit Care Med 2009;37:2740-5.

19 Jolley CJ, Luo YM, Steier J, et al. Neural respiratory drive in healthy subjects and in COPD. Eur Respir J 2009;33:289-97.

20 Piquilloud L, Vignaux L, Bialais E, et al. Neurally adjusted ventilatory assist improves patient-ventilator interaction. Intensive Care Med 2011;37:263-71.

21 Vignaux L, Grazioli S, Piquilloud L, et al. Patient-ventilator asynchrony during noninvasive pressure support ventilation and neurally adjusted ventilatory assist in infants and children. Pediatr Crit Care Med 2013;14:e357-64.

22 Murphy PB, Davidson C, Hind MD, et al. Volume targeted versus pressure support non-invasive ventilation in patients with super obesity and chronic respiratory failure: a randomised controlled trial. Thorax 2012;67:727-34.

23 Storre JH, Magnet FS, Dreher $\mathrm{M}$, et al. Transcutaneous monitoring as a replacement for arterial PCO(2) monitoring during nocturnal non-invasive ventilation. Respir Med 2011;105:143-50.

24 Carteaux G, Lyazidi A, Cordoba-Izquierdo A, et al. Patient-ventilator asynchrony during noninvasive ventilation: a bench and clinical study. Chest 2012;142:367-76.

25 Luo YM, Moxham J, Polkey MI. Diaphragm electromyography using an oesophageal catheter: current concepts. Clin Sci 2008;115:233-44.

26 Gandevia SC, Hudson AL, Gorman RB, et al. Spatial distribution of inspiratory drive to the parasternal intercostal muscles in humans. J Physiol 2006;573(Pt 1):263-75.

27 Janssens JP, Borel JC, Pepin JL, et al. Nocturnal monitoring of home non-invasive ventilation: the contribution of simple tools such as pulse oximetry, capnography, built-in ventilator software and autonomic markers of sleep fragmentation. Thorax 2011;66:438-45.

28 Hart N, Hunt A, Polkey Ml, et al. Comparison of proportional assist ventilation and pressure support ventilation in chronic respiratory failure due to neuromuscular and chest wall deformity. Thorax 2002;57:979-81.

29 Epstein SK. How often does patient-ventilator asynchrony occur and what are the consequences? Respir Care 2011;56:25-38.

30 Hull J, Aniapravan R, Chan E, et al. British Thoracic Society guideline for respiratory management of children with neuromuscular weakness. Thorax 2012;67(Suppl 1): i1-40.

31 Contal O, Adler D, Borel JC, et al. Impact of different back-up respiratory rates on the efficacy of non-invasive positive pressure ventilation in obesity hypoventilation syndrome: a randomized trial. Chest 2013;143:37-46.

32 Windisch $\mathrm{W}$, Haenel $\mathrm{M}$, Storre $\mathrm{JH}$, et al. High-intensity non-invasive positive pressure ventilation for stable hypercapnic COPD. Int I Med Sci 2009;6:72-6.

33 Colombo D, Cammarota G, Alemani M, et al. Efficacy of ventilator waveforms observation in detecting patient-ventilator asynchrony. Crit Care Med 2011;39:2452-7.

34 Mulqueeny Q, Redmond SJ, Tassaux D, et al. Automated detection of asynchrony in patient-ventilator interaction. Conf Proc IEEE Eng Med Biol Soc 2009;2009:5324-7.

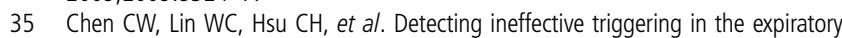
phase in mechanically ventilated patients based on airway flow and pressure deflection: feasibility of using a computer algorithm. Crit Care Med 2008;36:455-61. 